\section{Cahiers de Narratologie}

Analyse et théorie narratives

$16 \mid 2009$

Images et récits

\title{
Le langage des couleurs dans les films de Zhang Yimou
}

\section{Xiaomin Giafferri}

\section{(2) OpenEdition}

Journals

Édition électronique

URL : http://journals.openedition.org/narratologie/982

DOI : 10.4000/narratologie.982

ISSN : 1765-307X

Éditeur

LIRCES

Référence électronique

Xiaomin Giafferri, «Le langage des couleurs dans les films de Zhang Yimou », Cahiers de Narratologie [En ligne], 16 | 2009, mis en ligne le 25 mai 2009, consulté le 19 avril 2019. URL : http:// journals.openedition.org/narratologie/982 ; DOI : 10.4000/narratologie.982

Ce document a été généré automatiquement le 19 avril 2019

\section{(c) (i) (9)}

Cahiers de Narratologie - Analyse et théorie narratives est mis à disposition selon les termes de la licence Creative Commons Attribution - Pas d'Utilisation Commerciale - Pas de Modification 4.0 International. 


\title{
Le langage des couleurs dans les films de Zhang Yimou
}

\author{
Xiaomin Giafferri
}

1 Tout film, ou du moins tout film de fiction, est récit. Représentation visuelle et sonore, le film transpose à l'écran la réalité par un enchaînement d'images, où des traits physiques, des gestes, des mouvements et des paysages se combinent pour former un espace de fiction.

2 Si l'on admet que le cinéma en tant qu'art possède un langage spécifique et que les limites du récit filmique diffèrent de celles du récit verbal, romanesque par exemple, les couleurs participent-elles à la prise en charge de l'histoire ou font-elles simplement partie des jeux esthétiques? En effet, tout comme la troisième dimension du film dont l'absence ne gêne pas la reconnaissance visuelle, la couleur ne constitue pas, à priori, un trait pertinent d'identification.

3 Pourtant, en considérant les films de Zhang Yimou, connus depuis maintenant une vingtaine d'années de l'Occident, on ne peut s'empêcher de poser cette question : l'histoire racontée dans Le sorgho rouge, Epouses et concubines, Judou ou encore Hero auraitelle été la même si le film avait été en noir et blanc? Composante de l'image, qui ellemême est composante de l'espace filmique dont les fonctions narratives sont incontestées, la couleur est-elle définitivement non diégétique?

4 A partir de ces quatre films représentatifs aussi bien de l'art de Zhang Yimou que du nouveau cinéma chinois ${ }^{1}$, notre première réflexion portera sur le statut de la couleur dans un récit filmique, avant de tenter, dans un deuxième temps, de voir les spécificités de ses fonctions dans les récits de Zhang Yimou à travers notamment les films que nous venons de citer.

5 L'expression « récit filmique », selon André Gardies, fournit la parfaite illustration d'un embarras théorique lié au problème de la spécificité. Il souligne les deux dimensions qu'elle inclut et la question de la priorité: en privilégiant le "récit ", la spécificité du médium s'efface et le film n'est qu'une des manifestations possibles; en mettant le 
«filmique » en premier, la dimension langagière du médium l'emporte sur la fonction narrative.

6 Avec la notion de la «narrativité », on sort de l'embarras. Le récit est avant tout un ensemble d'événements. Cette vision rejoint la définition de Claude Bremon, pour qui le récit se caractérise par le fait qu'il est « indépendant du médium qui le prend en charge ${ }^{2}$ ", alors que la narrativité se définit par son caractère virtuel qui n'a d'existence qu'une fois actualisée dans un discours narratif.

7 Classée selon cette optique, la couleur comme composante du matériel visuel est, comme la troisième dimension du cinéma, non narratif-représentatif. Elle ne participe pas à l'agencement de thème et, de ce fait, ne constitue pas un trait constant de la narrativité. Le spectateur, habitué à la présence de la fiction, a en effet toujours tendance à la réinjecter dans l'image et à établir une relation entre les plans et les éléments; n'importe quelle couleur, dans ce cas, aurait servi d'embrayeur de fiction.

8 Mais le récit, c'est aussi le lieu de la rencontre contenu-expression, définie par Marc Vernet comme « l'énoncé dans sa matérialité ${ }^{3}$ ». Le récit filmique étant du film modélisé par la narrativité en même temps qu'il la met en forme, la couleur dans un film coloré est un des moyens d'expression qui participe, malgré son « a-narrativité », à « l'organisation interne $d u$ sens du film». Le caractère iconique du signifiant filmique lui consigne certaines valeurs, qui font de la couleur aussi un médium dont est tributaire la narrativité de la fiction.

9 La valeur cognitive de la couleur consiste à rendre compte des informations narratives et des actions mises en scène en examinant leur pertinence pour la compréhension de l'histoire. Il s'agit, hormis la question esthétique, d'introduire dans les éléments du décor les signes qui représentent l'espace filmique.

10 L'espace filmique comme cadre offert à l'action n'est pas un support abstrait, il est construit en fonction des nécessités de la "mise en scène» du contenu figuratif. La couleur n'y est pas indispensable, certes, mais en tant que composante de l'image, elle a une valeur d'évocation plus qu'une simple valeur de présence.

Les films de Zhang Yimou mettent souvent en œuvre une focalisation spectatorielle, afin de donner un avantage cognitif au spectacle sur des vues et une intensité de la représentation de la réalité. Les éléments du décor et les costumes des personnages se chargent de la localisation spatiale, les couleurs, loin d'être choisies arbitrairement, renforcent l'effet de réel et situent l'action dans un contexte, celui de la Chine traditionnelle. Du moment que le signe iconique se caractérise par les traits de ressemblance qu'il entretient avec l'objet qu'il désigne, une trace visuelle de la couleur, bien que non essentielle, a une valeur indicielle.

12 Mais les couleurs représentées ont une force évocatrice qui dépasse même sa fonction de reconnaissance, car un film n'est pas seulement un échantillon de cinéma, mais aussi un échantillon de culture. Dans les films de Zhang Yimou, les couleurs confèrent à l'espace diégétique une vérité, conformément à l'image culturellement attendue. Le rouge, par exemple, couleur des noces et du mariage, est omniprésent dans Epouses et concubines, il apparait aussi dans Le Sorgho rouge et dans Judou pour suggérer à lui seul l'union d'un homme et d'une femme, sans autre mention verbale et en dehors de toute image qui y fait allusion. Dans Hero, la couleur des tenues va jusqu'à constituer un indice sur les relations des personnages. Les deux héroïnes qui cherchent à aider le héros dans son projet initial de tuer le roi du Qin, le futur premier empereur de Chine, s'habillent toutes les deux en 
rouge mais les teintes sont nuancées : l'une porte un rouge vif tandis que l'autre toujours un rouge dégradé en rose ou en rouge pâle, couleur traditionnellement destinée à une concubine en présence de l'épouse principale. Ce détail, pour le spectateur culturellement initié, explique une certaine rivalité remarquée entre la compagne du héros et sa servante qui lui voue une passion.

Quand l'indice devient élément diégétique du drame, la représentation n'est-elle pas aussi narration?

La double caractéristique (iconicité et indicialité) font que la couleur assure une fonction qui consiste d'abord à montrer. Mais l'acte de monstration est un acte langagier que le cinéma utilise pour raconter. Dans l'espace qui est le point de rencontre de la technique et de l'esthétique, la couleur est un moyen d'expression où le langage et l'art sont cosubstantiels.

Pour Zhang Yimou, la couleur est un des moyens pour introduire d'emblée le monde exprimé et l'univers du connoté. Il est vrai que dans ses films plus récents comme Le Secret des poignards volants ou encore La Cité interdite, les couleurs ont plutôt valeur ornementale et sont dramatiquement neutres, dans un souci de donner du spectacle, mais dans la plupart des films de Zhang Yimou, notamment ceux de la période "réaliste », elles ne sont jamais composantes purement plastiques ou pittoresques d'un cadre.

Selon André Gardie, l'auteur du Récit filmique, la fonction narrative de la monstration, qui n'est pas de nature, résulte d'un ensemble de décisions. « Décisions qui relèvent, bien sûr, du réalisateur mais supposent aussi une compétence interprétative du spectateur. C'est probablement là, dans ce double jeu croisé du réalisateur et du spectateur, que se développe la performance narrative du cinéma ${ }^{4} . .$. »

Chez Zhang Yimou, cet ensemble de décisions tient de la solidarité des éléments concertés. Le rouge, couleur le plus souvent mise en scène, est le symbole de la femme selon la tradition chinoise. Dans Epouses et concubines, il s'associe à un objet, la lanterne, pour créer un cadre où l'ambiance dramatique est tributaire de l'effet d'authenticité. Dans Judou où le cadre de l'action est une teinturerie, les différentes couleurs du tissu étendu occupent tour à tour l'écran et donnent, à travers la nature sensorielle de chaque couleur, une émotion propre à chaque situation diégétique.

Dans Hero, où le récit est divisé en plusieurs séquences narratives distinctes, la manipulation de la couleur au service du déroulement du récit est la preuve de sa double présence: au plaisir de l'histoire, s'ajoute le plaisir lié au médium cinématographique. Parfois même, le réalisateur s'en sert pour l'organisation du récit filmique. L'histoire dans ce film se déploie à travers une conversation entre le roi du Qin, le futur premier empereur de Chine et Wuming, vainqueur des trois maîtres d'arts martiaux qui s'étaient jurés de tuer le roi afin de sauver la paix du monde. Grâce à cet exploit, Wuming est reçu par le roi comme celui-ci l'avait promis. Plusieurs séquences méta-diégétiques présentent alternativement le récit de Wuming sur les circonstances de la mort des trois maîtres et l'interprétation du roi ; chaque séquence est filmée sous un fond de couleur dominante, si bien que l'on peut les qualifier respectivement de récit rouge, récit bleu, récit blanc et récit vert. Dans le premier des quatre, représenté sous un ton rouge, Wuming raconte comment il avait tué Epée Ancien, l'ennemi le plus dangereux du roi. Les armes, les bannières, les habits de personnages et le décor du temple où les deux hommes se sont mesurés sont en rouge, ainsi que le signe calligraphié, symbole de la paix, montré plusieurs fois en gros plan. Le rouge ne se confond d'ailleurs, durant toute la séquence, 
qu'avec le noir, qui rappelle la couleur fétiche de la dynastie Qin. Le récit «bleu » qui vient en deuxième redouble la même séquence temporelle pour présenter la version du roi de la même histoire. Epée Ancien, selon lui, se serait fait volontairement battre par Wuming pour lui permettre de s'approcher du roi. La séquence contient au total une quarantaine de plans successifs, tous dans des différents tons de bleu, c'est presque un micro film bicolore. Le récit «blanc » qui suit révèle la dernière volonté d'Epée Ancien: convaincu que la réunification sauvera la Chine, il persuade Wuming de renoncer à tuer le roi. Le dernier des quatre micros récits, le " vert ", montre l'affrontement entre Wuming et Fleur de Neige, une autre ennemie jurée du roi qui voulait venger sa famille ; la mort de l'héroïne a déjoué l'ultime plan d'assassina contre le roi. Le choix esthétique des couleurs, autant que l'acte de la narration, joue ainsi au niveau de l'énonciation.

Lorsque la mise en scène des couleurs manifeste une volonté évidente de raconter, comme dans les films que nous venons de citer, la monstration devient un acte de narration. Les diverses stratégies de Zhang Yimou donnent à voir certaines fonctions qui procurent à la couleur des valeurs spécifiques.

Le cinéma est apte à vaincre l'espace, en nous transportant en un instant à n'importe quel point de la planète. L'expressivité du cinéma obéit à un mécanisme sémiologique: le sens se dégage de l'ensemble du signifiant, aucun élément du décor n'est gratuit. La couleur, moyen d'expression afilmique, devient un objet manipulé, une fois filmée, découpée et agencée selon une intention bien précise, elle possèdera son expressivité propre. Chez Zhang Yimou, par-delà une fonction de connaissance, la couleur contribue à faire apparaître des espaces dramatiques grâce à l'ensemble des manipulations concertées et à son agencement dans le récit.

21 D'abord, l'importance de la couleur dans la narration est souvent soulignée par le titre, comme le montre Le sorgho rouge, mais aussi Epouses et concubines, dont le titre original est Les lanternes rouges sont suspendues. La couleur rouge est visiblement choisie pour apparaitre comme l'une des grandes figures de la narration, elle fait l'objet d'une focalisation privilégiée dès le début du film. Le titre en idéogrammes rouges, affiché sur un fond noir, fait penser à la passion dévastatrice. Ce procédé, de même que l'alternance du rouge et du noir, autre couleur préférée du réalisateur, revient dans trois films: Epouses et concubines, Judou et Hero, tous les trois ont en leur titre en rouge sur le fond noir. Les deux couleurs denses et vigoureuses, par opposition à des teintes claires et gaies de certaines scènes, produisent un effet sensoriel bouleversant. Quant au Sorgho rouge, son titre paraît en un rouge plus lumineux et incandescent, l'association des deux mots qu'il contient évoque, pour le spectateur informé, le feu et la guérilla durant la guerre de résistance contre le Japon.

La lanterne rouge incarne l'objet du désir des épouses et concubines dans une vaste demeure, composée de multiples cours d'habitation, où son propriétaire, un riche dignitaire, vient d'accueillir sa quatrième épouse ${ }^{5}$. La lanterne qu'on allume chaque soir à la porte d'une des épouses est le signe de la faveur du maître; celle qui est choisie passe la nuit avec son mari, elle aura alors le droit de se faire masser et de commander des plats spéciaux. Pour avoir tous les soirs la lanterne, la quatrième épouse (incarnée par Gong Li, devenue dès lors une star) allait jusqu'à feindre une grossesse, et la bonne qui rêvait de devenir la cinquième épouse du maître décorait secrètement sa chambre avec des vieilles lanternes qu'elle avait récupérées. faire un leitmotiv. L'image de la cour, où toutes les épouses se tiennent debout sous 
l'éclairage de deux rangées de lanternes, est un tableau immobile qui revient jusqu'à 14 fois dans le film. La même scène apparaît aussi quelquefois sans la lumière colorée, la lanterne éteinte et masquée d'un tissu noir constitue alors un élément diégétique. La première apparition d'une telle scène intervient lors de la découverte de la fausse grossesse : l'ordre était donné de couvrir la lanterne de la tricheuse, la seconde a lieu après la mort de la bonne qui a enfreint l'interdiction d'allumer des lanternes dans sa chambre, et la troisième fois, elle annonce la mort de la troisième épouse, pendue pour avoir eu une liaison. La dernière image du film se fixe en zoom sur la même cour sans le rouge qu'on aperçoit au début du film, au milieu d'une masse de toits gris dominant la maison forteresse.

La présence et l'absence des lanternes rouges caractérisent ainsi une situation du programme narratif. Au lieu d'être réduite à sa seule enveloppe plastique, la couleur est porteuse du dynamisme qui régit le récit, elle entre dans un système d'échange avec le sujet. Entre le sujet et l'objet du désir s'installe une relation «jonctive», faite soit de conjonction, soit de disjonction. Le récit consistera dès lors à raconter la transformation de cette relation. Si dans le cinéma, la bande raconte, ici c'est la bande en couleur qui raconte. A défaut d'explication linguistique, un film en noir et blanc aurait-il donné le même sens?

Quand l'image entre en relation avec le spectateur dans un complexe affectif et intellectuel, remarque Marcel Martin, auteur du Langage cinématographique, "la signification qu'elle prenait à l'écran dépendait de l'activité mentale du spectateur presque autant que de la volonté créatrice du réalisateur ${ }^{6} \%$.

L'espace filmique étant un volume dramatique et non pas un environnement simplement descriptif, toutes les composantes des données figuratives et plastiques sont significatives. Les films de Zhang Yimou sont faits d'images qui prennent leur sens les unes par rapport aux autres, par un jeu d'implications réciproques, de symboles et d'ellipses, pour entrer en résonance avec l'intériorité des individus. Le sens profond de la réalité se fait par une suggestion plus que la simple perception du contenu apparent. Les films sont lisibles à plusieurs niveaux, selon le degré de sensibilité d'imagination et de culture du spectateur.

Du fait que la couleur est utilisée pour la conception des métaphores et des symboles, le moyen d'expression qui ne produit normalement qu'une variante non pertinente en dénotation, devient pertinente en connotation. La couleur permet à Zhang Yimou d'investir une valeur supplémentaire aux éléments de la fiction. Il s'agit des plans ou des scènes qui, tout en appartenant à l'action, apportent une signification plus profonde et plus large. La motivation des couleurs donne lieu à des conventionnalisations qui participent à signifier l'histoire racontée.

Comme nous l'avons vu à propos d'Epouses et concubines, le rouge de la lanterne, couleur du mariage traditionnel, est mis en avant par différentes techniques. La première nuit de la nouvelle quatrième épouse dans la demeure est une scène où tout apparaît sous une coloration rouge, suivie de l'image d'un lit; une scène similaire paraîtra encore plusieurs fois et la lanterne rouge suffira à suggérer une nuit de couple. L'élément diégétique s'enrichit dès lors d'une valeur symbolique qui lui est conférée par le jeu de contexte et le rapprochement d'image. Le spectateur averti fera sans doute une liaison avec Le sorgho rouge, où la couleur rouge a été aussi utilisée avec le même sens symbolique : l'acte d'amour d'un porteur de palanquin avec la mariée qu'il portait, forcée d'épouser un vieux lépreux, n'est pas représenté mais seulement suggéré à l'écran par l'image d'un champ de 
sorgho rouge. La scène, inondée de façon presque surréaliste par une lumière rouge est accompagnée d'une voix-off qui chante, les paroles de la chanson soulignent encore la couleur des habits de la mariée et de l'alcool de sorgho, l'interaction entre le visuel et le sonore renforce le sens symbolique.

Pour qu'une couleur choisie en arrive à donner un accroissement de sens à l'image perçue à l'écran, Zhang Yimou mobilise un ensemble de processus et de techniques pour sa représentation. Le rouge du mariage qui fait penser d'abord au couple et à la passion est évoqué également dans Judou, mais il constitue, grâce au sens symbolique qu'il porte, un indice important de la diégèse. On se sert, ici, de la juxtaposition des images dans l'esprit du spectateur. D'abord, l'image d'un lit, des draps et des couvertures rouges apprend que le teinturier vient de passer la nuit de noces avec sa nouvelle épouse. Une seconde scène identique, plus loin, montre la même femme avec un autre homme, le neveu du propriétaire et employé du teinturier. La scène est suivie d'une image symbolique, où un long tissu suspendu tombe subitement dans l'eau de teinture, rouge comme le sang. Le rapprochement des deux images produit ainsi dans l'esprit du spectateur un choc psychologique, on devine un inceste et on l'enregistre comme un élément de l'intrigue du récit.

De la même manière que la lanterne dans Epouses et concubine ou le champ de céréale dans Le sorgho rouge, les bandes de tissus inondent l'écran à plusieurs reprises. La chute du tissu dans l'eau rouge, par exemple, précède ou suit toujours un événement important dans le récit. Vers la fin du film, le fils né de la liaison illégale, jette son propre père biologique dans le bassin, pour venger son père légitime et sauver sa propre réputation, de longues bandes de tissu suspendues tombent l'une après l'autre, avant de se noyer dans la teinture rouge.

Une autre image qui revient à plusieurs reprises est celle des tissus rouges, orange ou jaunes sur un fond de toits noirs. Comme dans un tableau, ces couleurs vives tranchent sur un fond sombre. L'image apparaitt pour la première fois lors de la naissance du fils de la liaison illégitime, sa deuxième apparition a lieu quand la mère avoue à son mari que l'enfant n'est pas de lui, et la troisième correspond au moment où le garçon apprend la vérité. La répétition de l'image devient un indice, dont la signification se révèle au fur et à mesure.

Parfois, les couleurs ont tant de poids que les objets qui les portent en sont presque devenus de simples supports. Peut-on penser que le rouge a définitivement moins de valeur narrative et représentative que la lanterne, le sorgho ou le tissu?

Les connotations signifiées, dont l'origine a des causes diverses, fait appel non seulement à l'imagination et à la culture mais aussi à l'auto-parodie entre des films. Par une logique d'implication propre au cinéma, les images s'emboîtent les unes dans les autres pour faire sortir le contenu latent. Avec tout son bagage symbolique, le rouge fait allusion au mariage dans Epouses et concubines, à l'amour dans Le sorgho rouge et à l'inceste dans Judou. De même, la teinture rouge de Judou fait penser au crime commis dans la grande maison des épouses et concubines, et le champ de sorgho qui a vu tomber des résistants communistes aux héros qui se sont sacrifiés, il y a deux mille ans, pour l'oeuvre de la réunification de la Chine. L'usage des couleurs poussé jusqu'à la saturation fait ressortir leur signification latente, c'est ainsi que le rouge des noces du sang et du feu en arrive à traduire la passion, le meurtre, la guerre et la révolution. 
L'auto-parodie peut s'étendre même à d'autres films de Zhang Yimou, voire aux films d'autres cinéastes chinois de la même génération, comme Chen Kaige, réalisateur de L'empereur et assassin et d'Adieu, ma concubine, pour ne citer que des films connus du public européen. Par un jeu d'interférences et de références, la présence de l'objet choisi pour symboliser à l'écran suffit à évoquer un ensemble de significations implicites qui font valoir la couleur plus qu'elle-même et aboutir à des codes spécialisés.

Ceci est le résultat d'une focalisation privilégiée, à laquelle Zhang Yimou consacre divers moyens d'expression cinématographique. Montage, cadrage, profondeur de champ et effet optique sont mis en oeuvre pour les mettre en scène. La rhétorique de l'écran est utilisée de façon dramatique pour un effet sensoriel spécial. La superposition d'une couleur dominante à toutes les autres est l'un des procédés favoris du réalisateur. Dans Epouses et concubines, après avoir symbolisé la nuit conjugale, la lumière rouge remplit une chambre déserte, celle de la troisième épouse, pendue pour avoir eu une liaison. Dans Le sorgho rouge, le même procédé est utilisé six fois, l'écran devenu quasi monochrome reflète une vision irréelle: le ciel et la terre sont perçus à travers une lumière intensifiée par la couleur de l'alcool et du sang. La focalisation de ces images bénéficie d'une localisation privilégiée. Le début et la fin du film sont souvent l'endroit choisi pour renforcer l'effet. La dernière scène de Judou, par exemple, se termine par une métaphore similaire: le rouge du tissu qui dénote le meurtre du père par son propre fils se transforme en un feu incandescent, tandis que la voix d'une chanson déjà entendue au début du film rappelle le rouge du titre.

Ces couleurs subjectives ne traduisent pas l'état supposé objectif de la diégèse, mais la vision des personnages. Elles insèrent des images mentales au milieu d'une séquence qui fonctionne sur un autre plan de la réalité. La volonté picturale enlève la réalité au contenu diégétique, ce réel trop expressif devient irréel, et il n'est plus que signe.

La constatation de ces résultats nous permet de conclure que bien que la couleur existant dans le monde usuel ne soit pas récit, elle cesse d'être une réalité a-filmique ou un élément simplement socio-culturel lorsque le réalisateur fait d'elle un composant expressif qui s'articule de façon aussi signifiante à l'histoire. Le langage des couleurs de Zhang Yimou est à la fois mimique et diégétique, c'est un moyen d'écriture aussi subtil que le langage écrit et si vigoureux que la pensée peut se traduire en couleur sur la pellicule.

8 Si l'on croit l'idée à laquelle ont abouti des expériences filmologiques sur la mémoire du film, le spectateur ne retient d'un film que son intrigue et au mieux quelques images. Celles qui nous restent des films de Zhang Yimou seraient-elles dissociables des couleurs?

Tout film, ou du moins tout film de fiction, est récit. Représentation visuelle et sonore, le film transpose à l'écran la réalité par un enchaînement d'images, où des traits physiques, gestes, mouvements, paysages se combinent pour former un espace de fiction.

Le cinéma en tant qu'art possède un langage spécifique; dans un film coloré, les couleurs comme moyen d'expression font partie du langage pictural. A partir de quatre films de Zhang Yimou connus en Europe, Le sorgho rouge, Epouses et concubines, Judou et Hero, ce texte tente de réfléchir sur ces questions: si l'on admet que les limites du récit filmique diffèrent de celles du récit verbal, romanesque par exemple, les couleurs qui ne constituent pas un trait pertinent d'identification, participent-elle à la prise en charge de 
l'histoire? Composante de l'espace filmique dont les fonctions narratives sont incontestées, sont-elle définitivement non diégétiques?

\section{NOTES}

1. Nous entendons par cette expression le cinéma chinois qui sort de ses marques officielles et fait son entrée dans le monde.

2. Claude Bremon, Logique du récit, Paris, Seuil, 1973

3. Marc Vernet, Esthétique du film, Paris, Nathan, 1983

4. André Gardies, Le récit filmique, Hachette, Paris, 1993, p. 18-19

5. En Chine, la polygamie au sens strict est réservée à la population musulmane en conformité avec les règles de l'Islam, tandis que la tradition chinoise permet le concubinage: les concubines, qui se dit "qie", n'ont pas le même statut et ne bénéficie pas des mêmes droits que l'épouse principale, "qi".

6. Marcel Martin, Le langage cinématographique, Cerf, Paris 1994, p.103.

\section{RÉSUMÉS}

Tout film, ou du moins tout film de fiction, est récit. Représentation visuelle et sonore, le film transpose à l'écran la réalité par un enchaînement d'images, où des traits physiques, gestes, mouvements, paysages se combinent pour former un espace de fiction. Le cinéma en tant qu'art possède un langage spécifique; dans un film coloré, les couleurs comme moyen d'expression font partie du langage pictural. A partir de quatre films de Zhang Yimou connus en Europe, Le sorgho rouge, Epouses et concubines, Judou et Hero, ce texte tente de réfléchir sur ces questions: si l'on admet que les limites du récit filmique diffèrent de celles du récit verbal, romanesque par exemple, les couleurs qui ne constituent pas un trait pertinent d'identification, participent-elle à la prise en charge de l'histoire? Composante de l'espace filmique dont les fonctions narratives sont incontestées, sont-elle définitivement non diégétiques?

\section{INDEX}

Mots-clés : fiction, récit, film, couleur, langage pictural, chinois, Yimou Zhang, limites, filmique, narratif, diégétique 
AUTEUR

XIAOMIN GIAFFERRI

Université de Nice-Sophia Antipolis, CIRCPLES, EA 3159 